\title{
Zur Gründungsgeschichte der Schweizerischen Krebsliga (SKL) ${ }^{1}$
}

Stefan Hächler

\section{SUMMARY}

The Swiss Cancer League was founded in 1910 under the name of "Schweizerisches Komitee für Krebsforschung". It was initiated by the first director of the Swiss Federal Health Office, Dr. Friedrich Schmid. Schmid received most impulses from the Genevan "Société de la lutte contre le cancer", founded in 1907 in Geneva. Its mentor, Dr. Robert Odier, had struggled for federal support and for membership in the International Organisation for Cancer Research (founded in 1908 in Berlin). The main purpose of the "Schweizerisches Komitee für Krebsforschung" was to start a national cancer research program. For this reason, another society was founded, the "Schweizerische Vereinigung für Krebsforschung». It had to procure money and provide information of lay people and physicians as well. During and after World War I activities stagnated and reorganisation became necessary. Changes of personnel, new activities and topics and opening membership to non-medical persons initiated a new phase of modernization.

\section{ZuSAMMENFASSUNG}

Die Geschichte der Schweizerischen Krebsliga beginnt am 1.5.1910, als sich unter dem Vorsitz des damaligen Direktors des eidgenössischen Gesundheitsamtes ( heute BAG), Dr. Friedrich Schmid, ein ärztliches «Schweizerisches Komitee für Krebsforschung» bildet. Vorausgegangen waren vergebliche Bemühungen der 1907 von Dr. Robert Odier gegründeten Genfer "Société de la lutte contre le cancer» um Bundessubventionen und um Anschluss an die "Internationale Vereinigung für Krebsforschung»( IVK, 1908 in Berlin ins Leben gerufen). Das «Schweizerische Komitee für Krebsforschung» setzt sich zum Ziel, eine «Schweizerische Vereinigung für Krebsforschung» (SVK) zu gründen, die in erster Linie die Mittel zu einem schweizerischen Krebsforschungsprogramm 
beschaffen sollte. Nach anfänglichen Schwierigkeiten der Mitglieder-Rekrutierung und unterschiedlichen Ansichten betreffend die wissenschaftliche und thematische Ausrichtung der SVK beginnt bald eine rege Tätigkeit, zuerst v.a. auf dem Gebiet der Öffentlichkeitsarbeit (Sensibilisierung von Fachleuten und Bevölkerung), bald auch auf den Gebieten von Forschung und Praxis. Der Erste Weltkrieg hemmt die Aktivitäten erst um 1918. Personelle Erneuerungen, neue Erkenntnisse und Akzentverschiebungen in den Aktivitäten geben der SVK ab ca. 1920 ein neues, moderneres Gepräge. Sie verlässt das Gründungsstadium und tritt in eine Konsolidierungsphase.

«Entreprendre l'organisation d'une campagne contre le cancer dans un pays, implique avant tout d'entreprendre celle contre les préjugés (...)»

(Robert Odier, 1925)

\section{Einleitung}

Zwar hat schon Wegelin ${ }^{2}$ über die Geschichte der SKL geschrieben, aber die Gründung und die ersten zehn Jahre des Bestehens handelt er nur knapp ab. Zudem fehlt die Vorgeschichte fast ganz, das Wenige darüber ist nicht immer ganz zutreffend. Es soll deshalb hier versucht werden, einerseits die Gründung der Schweizerischen Krebsliga (SKL) ${ }^{3}$ in einen weiteren (medizin)historischen Kontext zu stellen, andererseits auch die Vorgeschichte und die Gründung anhand von (von Wegelin nicht verwendetem) Archivmaterial aus dem Archiv der SKL und aus dem im Bundesarchiv deponierten Material des damaligen Eidg. Gesundheitsamtes (EGA) nachzuzeichnen.

Es wird versucht, die SKL in ihren Ursprüngen im medizinischen und gesellschaftlichen Entwicklungsprozess einzuordnen. Allerdings kann das nur andeutungsweise geschehen. Eine präzisere Einordnung würde weitergehende Studien voraussetzen.

Nur gestreift werden konnte die internationale Entwicklung der Krebsforschung und -bekämpfung und die Stellung der Schweizerischen Vereinigung zur Krebsbekämpfung (SVK) innerhalb dieser Entwicklung. Ebenfalls 
interessant wäre sicherlich ein Kapitel über die Auseinandersetzung verschiedener medizinischer Richtungen und Methoden (z. B. Radiumtherapie) innerhalb der SVK gewesen. Es hätte vermutlich erlaubt, die SVK und ihre Mitglieder noch genauer in den medizinhistorischen Kontext einzuordnen.

\section{Die Krebsforschung bis ca. 1920}

Zum Verständnis der Anfangsbedingungen und -voraussetzungen der Gründung der Schweizerischen Krebsliga ist es wichtig, sich des beträchtlichen Spannungsverhältnisses innerhalb der Medizin und speziell der Tumormedizin zwischen zahllosen Theorien, zwischen experimentellen, diagnostischen und therapeutischen Ansätzen bewusst zu werden.

«The history of the therapy of cancer is very dull. The principles we are following today, namely, the elimination of the tumor as radically as possible, were discovered in far remote antiquity. Our operative methods are much more efficient than theirs were, and besides the knife we have X-rays and radium to destroy the tumor cells, but we have not found any new principles yet.» 4

Sigerists Zitat weist auf ein grundlegendes Problem der modernen Krebsforschung hin. Obwohl das Wissen um den Krebs im 19. und 20. Jahrhundert sprunghaft gestiegen ist, ist es weiterhin nicht möglich, jede Art von Krebs zu heilen. Ziel dieses Kapitels ist es, die Grundzüge der Entwicklung der Krebsforschung im 19. und frühen 20. Jahrhundert nachzuzeichnen, deren Bestreben es war (und auch heute noch ist), das erwähnte Grundproblem zu lösen. Dabei muss exemplarisch bleiben, was in der medizinischen Literatur schwer übersehbar erscheint.

\section{Allgemein}

Die Anfänge der modernen Krebsforschung entspringen den Erkenntnissen, die aus den neuen Disziplinen histologische Pathologie und Zellpathologie erwuchsen. 1802 entdeckte Bichat, dass der menschliche Körper nicht nur aus verschiedenen Organen zusammengesetzt ist, sondern dass er auch aus verschiedenen Geweben besteht. Die von Schleiden und Schwann 1837/38 formulierte Zelltheorie und der Einsatz immer besserer Mikroskope eröffneten der Krebsforschung neue Horizonte. Endlich konnte die schon lange 
behauptete lokale Ursache des Krebses an einem bestimmten Ort gesucht werden. Die Zelle wurde das bevorzugte Untersuchungsobjekt von Krebsforschern. Johannes Müller suchte und fand schon 1838 die «Tumorzelle» und bestimmte damit eine Hauptrichtung der modernen Krebsforschung ${ }^{5}$. Die anatomisch-pathologisch-histologische Wissenschaft vom Krebs gewann durch Rudolf Virchows «Cellularpathologie» und deren Anwendung auf Tumore sogar zentrale Bedeutung im 19. und auch im 20. Jahrhundert. Virchows radikale Verfechtung der Lokalität des Krebses (Hauptursache für Geschwulstbildung war nach Virchows Ansicht eine lokale Reizung) und seine Ansicht, dass Geschwülste «nur» eine abnormale Zellvermehrung seien, ebneten den Chirurgen ein weites Betätigungsfeld ${ }^{6}$ und etablierten deren faktische Monopolstellung bei der Krebstherapie. Auf diesem Hintergrund entstanden in der zweiten Hälfte des 19. Jahrhunderts diverse Forschungsrichtungen und -meinungen zum Krebs. Aber der auf Müller und Virchow zurückgehende Grundkonsens in der sog. «Schulmedizin» (vielleicht wäre der Begriff «Paradigma» angebracht) wurde nicht ernstlich gefährdet $^{7}$. Im wesentlichen ging es in den nächsten Jahrzehnten darum, Virchows Geschwulsttheorie zu verfeinern, unhaltbare Annahmen zu korrigieren und neue Aspekte beizufügen ${ }^{8}$.

Eine erste wesentliche Korrektur erfuhr Virchows These, wonach alle Geschwülste aus dem Bindegewebe entstünden. Remak, Hannover, Michel, Tiersch und Waldeyer bewiesen in den 1850er und 60er Jahren, dass die Tumoren aus dem Epithel entstehen. In der Folge wurde die morphologische Geschwulstforschung ein wichtiger Aspekt. Dank der Feinerfassung des geweblichen Formenreichtums der Geschwülste konnte die histologische Geschwulstdiagnostik präziser, schneller und erfolgreicher arbeiten ${ }^{9}$. Ein Schüler Virchows, Cohnheim, stellte der Reiztheorie Virchows als Ursache der Geschwülste das Konzept der gestörten Entwicklung entgegen, welches Geschwülste als «Verlagerungen und Ausschaltungen während der embryonalen Entwicklung» (der Zellen) ${ }^{10}$ erklärt. Dagegen wurde die Reiztheorie am Ende des Jahrhunderts dahingehend weitergeführt, dass Geschwülste in der Folge eines Regenerationsprozesses entstehen (Fischer-Wasels). Weitere Theorien über die Ursachen von Geschwülsten kamen im Zusammenhang mit neueren Disziplinen auf, die anatomisch-histologische Forschung wurde durch biologische, chemische und biochemische konkurrenziert (beispielsweise die durch die Bakteriologie genährte These des Krebsbazillus oder die aufgrund der Genetik postulierte Erbanlage zu Krebs). Die Ergebnisse der experimentellen Geschwulstforschung, die anfangs unseres Jahrhunderts 
eine wahre Blüte erlebte (mit den grundsätzlich drei Methoden der Geschwulstüberpflanzung, Geschwulsterzeugung und Gewebszüchtung), ermöglichte die bessere Überprüfung der wie Pilze aus dem Boden schiessenden Theorien, Subtheorien, Thesen, Konzepte und Vermutungen. Verwerfungen, Relativierungen, Einordnungen und Neuformulierungen von Theorien zur Entstehung der Geschwülste waren die Folge. Auf dem Gebiet der Therapie waren die Möglichkeiten der Chirurgie bald erschöpft. Hier galt es um die Jahrhundertwende, Operationstechniken und -methoden zu verfeinern und dank der verbesserten Diagnosemöglichkeiten möglichst früh zu handeln. Am Ende des 19. Jahrhunderts traten mit den Errungenschaften der Technik wie Elektrizität und Röntgenstrahlen ${ }^{11}$ neue Hoffnungen auf die Therapiebühne, die viele Erwartungen weckten und Impulse vermittelten, aber auch Spannungen erzeugten, da sich die Chirurgen plötzlich einer ungewohnten Konkurrenz gegenüber sahen.

\section{In der Schweiz}

Wie präsentierte sich nun aber die Krebsforschung den Schweizer Ärzten, die 1910 an der Gründungsversammlung des Schweizerischen Komitees für Krebsforschung teilnahmen? Einen Eindruck davon vermittelt uns der an der Gründungsversammlung von Dr. Max Oskar Wyss ${ }^{12}$ gehaltene Vortrag «Über den gegenwärtigen Stand der Krebsforschung». Wyss spricht zuerst der Cohnheimschen Theorie nur für wenige Geschwülste Gültigkeit zu. Tierschs Ansicht (erst wenn das Bindegewebe alt und schwach ist, können die Epithelzellen ungehindert wachsen) sieht er als kaum mehr geltend an. Mit Ribbert ist er der Überzeugung, dass die Initiative zum ungehinderten Wachstum von den Epithelzellen selbst ausgeht, wenn diese aus dem Gewebe- oder Organverband herausgelöst sind. Nachdem er seine Position geklärt hat, referiert er mehr oder weniger urteilsfrei über neueste Forschungen, Theorien und Diskussionen. So erläutert er Hansemanns Anaplasie (... «worunter eine Vereinfachung ihres [der Krebszelle] Baus durch Verlust an Differenzierungsvermögen zu verstehen ist.» ${ }^{13}$ ), führt die Ansätze von parasitären und Infektions-Theorien an (wobei er ihnen kaum Erklärungskraft zubilligt), zeigt die neuen Richtungen der Reiztheorien und die Weiterentwicklung der Cohnheimschen Theorie auf, weist auf neue Experimente hin, deren Resultate zu interpretieren sind, äussert sich skeptisch gegenüber neuen Therapieformen wie Elektro-, Radium-, Röntgen-, Chemo-, Serum-, Biotherapien, die bis dahin noch keine 
oder nur geringe Erfolge aufweisen konnten und hebt am Schluss die Bedeutung einer guten, sehr differenzierten und einheitlichen Statistik zur Ausrichtung der künftigen Krebsforschung hervor.

Wyss belegt mit seinem Referat, dass die Schweizer Ärzte sich auf dem aktuellen Informationsstand befanden. Auch in der Forschung konnte die schweizerische Medizin dank etlichen Ärzten, die sich in ihren Spezialgebieten mit onkologischen Problemen konfrontiert sahen und diese angingen, international mithalten, u. a. weil die Chirurgie und benachbarte Fachgebiete in der Schweiz ein beachtliches Niveau hielten. Die im internationalen Vergleich relativ hohe Krebssterblichkeit der Schweiz ${ }^{14}$ ist sicher ein weiteres Element, das die Auseinandersetzung mit dieser Krankheit begünstigte. Die zahlreichen Verbindungen mit ausländischen Krebsfachleuten ${ }^{15}$, die durch Studiensemester im Ausland, durch allgemeine und spezielle Ärztekongresse und durch die relative Kleinheit der medizinischen «scientific community» entstanden waren, mögen ebenfalls ihren Teil dazu beigetragen haben. Jedenfalls wurden im «Correspondenzblatt für Schweizer Ärzte» zwischen 1870 und 1900 über 300 Artikel, die von Krebs handeln, veröffentlicht ${ }^{16}$. Dass das Schwergewicht dabei auf der chirurgischen Behandlung (v.a. Exstirpation) diverser Tumore lag, erstaunt wenig. Besondere Beachtung fanden die Tumore des Verdauungsapparates und der weiblichen Geschlechtsorgane ${ }^{17}$. Gerade in dieser Beziehung ragten einige Schweizer Ärzte oder in der Schweiz lehrende Ausländer hervor. Erwähnt seien Theodor Kocher, Heinrich Bircher, August Socin, Ulrich R. Krönlein und auch Billroth, der etliche Jahre in Zürich wirkte. Daneben publizierten viele andere Ärzte (mit und auch ohne nationale bis internationale Reputation) über Krebs ${ }^{18}$. Allerdings fanden diese Forschungen weitgehend im Rahmen schon bestehender Institutionen statt, allenfalls gab es an bestimmten Instituten und Kliniken Unterabteilungen, die sich mit Krebs zu beschäftigen hatten, wie die Tumorenstation des Pathologischen Instituts der Uni Zürich, die 1900 von Prof. Dr. P. Ernst gegründet wurde ${ }^{19}$. Es gab aber bis nach der Jahrhundertwende keine eigenständigen Einrichtungen, ob öffentlich oder privat, die sich ausschliesslich der Krebsforschung oder der Krebsbekämpfung gewidmet hätten. Auch eine spezielle gemeinsame Plattform der Krebsforscher (z. B. in Form einer Zeitschrift) existierte nicht. Rosselet charakterisiert den Kampf gegen den Krebs zu dieser Zeit als «efforts isolés des chirurgiens» ${ }^{20}$, die allein auf persönlichem Engagement beruhten.

Vor diesem Hintergrund ist die Gründung der Schweizerischen Krebsliga zu sehen. 


\title{
Entstehung der Krebsliga
}

\author{
Vorgeschichte: Genfer Aktivitäten
}

Die ersten Anstrengungen, den Kampf gegen den Krebs in der Schweiz zu organisieren, wurden in Genf unternommen. In einer von Dr. Robert Odier ${ }^{21}$ am 15. April 1907 veranstalteten Konferenz über den Stand der Krebsbekämpfung zu Beginn des 20. Jahrhunderts wandte sich dieser in einem Referat gegen das (seiner Ansicht nach nicht nur unter Laien, sondern auch unter zahlreichen Ärzten) verbreitete Vorurteil, gegen den Krebs sei die Medizin machtlos.

\begin{abstract}
«Grâce aux progrès de la chirurgie, grâce aussi à la radiothérapie, nombreux sont les cancers que nous pouvons, plus nombreux encore ceux que nous pourrions guérir. Par des soins spéciaux et persévérants, que d'existences pourrions-nous améliorer, que de souffrances et de misères nous saurions épargner. Par une prophylaxie raisonnée dont un enseignement judicieux et proportionné aux diverses classes serait le principal agent, à combien de malades ne réussirions-nous pas à éviter désormais un mal abominable» ${ }^{22}$.
\end{abstract}

Odier verurteile die Lethargie und den Fatalismus, mit denen die grosse Mehrheit des Volkes und der Mediziner in der Schweiz dem Krebs gegenüberstünden. Dabei treffe die Krebskrankheit immer mehr junge Leute im besten Alter, "(...) où ils représentent pour la société le maximum de leur valeur

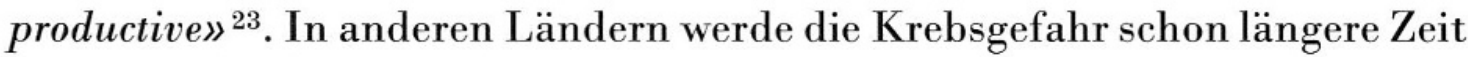
ernst und die Chancen der neuesten medizinischen Forschung wahrgenommen. In der Schweiz hingegen "(...) le péril cancéreux (...) est l'un de ceux contre lesquels on a le moins lutté en Suisse jusqu'ici et avec lequel il faut le plus compter aujourd'hui.» ${ }^{24} \mathrm{Um}$ dieser Misere abzuhelfen, regte er an, die Krebsforschung in Genf zu fördern :

«Il serait notamment déraisonnable pour le moment de chercher à créer ici un hôpital pour les cancéreux, mais par contre il est désirable d'encourager et de faciliter l'étude des méthodes autres que la chirurgie, la radiothérapie et les traitements électriques offrant quelques chances, sinon la guérison radicale, du moins d'amélioration et de soulagement aux souffrances de cette catégorie de malades.» ${ }^{25}$

Damit deutete Odier auch schon die von ihm gewünschte Richtung der Krebsforschung an. Nicht primär die chirurgischen Methoden sollten Gegenstand der künftigen Forschung sein, sondern andere Therapieformen wie Radio- und Elektrotherapie. Konkret wünschte er ein «(...) laboratoire 
consacré exclusivement à l'étude des moyens practiques de combattre le cancer», das von interessierten Personen finanziell getragen werden sollte ${ }^{26}$.

Noch im selben Jahr, 1907, fruchteten Odiers Vorschläge, indem eine Genfer «Société de la lutte contre le cancer» ${ }^{27}$ gegründet wurde, die ihrerseits im selben Jahr das «Institut pour l'étude des maladies cancéreuses» ins Leben rief. (Mit-?)Begründer beider Institutionen und erster Direktor des neuen Instituts war - Dr. Robert Odier ${ }^{28}$. Das Ziel der «Société» war laut Statuten die finanzielle Unterstützung von Krebsforschungslabors und die Umsetzung geeigneter Mittel der Prävention und Heilung von Krebskrankheiten in die Praxis ${ }^{29}$. Das «Institut» sollte zum Zweck haben, die verschiedenen Formen von Krebs zu studieren, Behandlungsmethoden zu prüfen und die Öffentlichkeit über die Krebskrankheiten aufzuklären ${ }^{30}$. Zu diesem Zweck veranstalteten Institut und Société (eine klare Abgrenzung zwischen beiden Institutionen ist kaum auszumachen) unter anderem auch Konferenzen mit internationalen Krebsfachleuten (u. a. mit Dominici und KaetingHart) und jährlich öffentliche Vorträge ${ }^{31}$. Daneben organisierten Mitglieder der Société auch in eigener Regie Veranstaltungen zum Thema Krebs ${ }^{32}$. Auch wurde eine Zeitschrift «Revue suisse du Cancer» an das interessierte Publikum verteilt. Des weiteren wurden Plakate eingesetzt, um breite Bevölkerungsschichten anzusprechen ${ }^{33}$.

In der Genfer Société waren 1908 folgende Männer im Vorstand aktiv ${ }^{34}$ : Dr. Edmund Lardy (Präsident) ${ }^{35}$, Dr. Robert Odier ${ }^{36}$, Prof. Dr. Charles Bergalonne ${ }^{37}$, Maurice Hentsch (Kassier), Prof. Dr. Adolphe d'Espine ${ }^{38}$, Prof. Dr. Duparc, Prof. Dr. Charles Girard ${ }^{39}$, Prof. Dr. J. L. Reverdin ${ }^{40}$ und Prof. Dr. Léon Massol ${ }^{41}$. Ausser dem Kassier Hentsch, der Bankier und Duparc, der Mineraloge war, bestand der Vorstand nur aus Ärzten.

Als im Juli 1908 in Berlin die «Internationale Vereinigung für Krebsforschung» (IVK) gegründet wurde, suchte auch die Genfer «Société» um Aufnahme nach. Die Internationale Vereinigung lehnte das Gesuch ab, weil sie nur nationale und keine regionalen Sektionen als Mitglieder aufnahm. Allerdings zeigte sich die Internationale Vereinigung interessiert an einer Schweizer Mitgliedschaft und verfasste am 1.12.1908 auf Veranlassung eines «Herrn Geheimen Oberstudienrats Professor Dr. Kirchner, vortragenden Rat im Ministerium der geistlichen, Unterrichts- und Medizinalangelegenheiten» in Berlin, einen Brief an den Direktor des schweizerischen Gesundheitsamtes, Dr. Schmid ${ }^{42}$, in dem sie ihn bat,

«... gütigst Schritte zu tun, um die Begründung eines gemeinschaftlichen Schweizerischen Komitees mit Mitgliedern aus allen Teilen des Landes, besonders aber auch mit den 
genannten Herren des Genfer Komitees, in die Wege zu leiten, damit dann dieses Schweizerische Komitee Mitglied der Internationalen Vereinigung für Krebsforschung werden kann.» ${ }^{43}$

Aus einem Brief von Dr. R. Odier vom Genfer Komitee an Dr. Schmid geht hervor, dass die Internationale Vereinigung erst auf ein Rekursschreiben aus Genf an das schweizerische Gesundheitsamt gelangt war. Dr. Odier unterstützte Bestrebungen zur Gründung eines schweizerischen Komitees vor allem zwecks Einritt in die Internationale Vereinigung, bestand aber auf einer Vertretung aus Genf, weil das Genfer Komitee «(...) le premier, s'est occupé de doter notre pays d'un Institut pour l'Etude du Cancer.» ${ }^{44}$

Aber nicht nur die Absicht, der IVK beizutreten, dürfte für die Genfer Motiv gewesen sein, eine gesamtschweizerische Organisation zu gründen. Wie aus einem Brief der Bundeskanzlei an das «Comité de l'Institut anticancereux» in Genf ${ }^{45}$ vom 9. März 1909 hervorgeht, ersuchte dieses am 2.12.1908 (wenige Tage vor der Bitte an Dr. Schmid um Gründung einer schweizerischen Vereinigung für Krebsforschung!) den Bundesrat um einen jährlichen Subventionsbeitrag von Fr. 10000 .-. Auch dieses Gesuch wurde mit Verweis auf die nur lokale Tätigkeit abgewiesen. Die Subventionsfrage könne allenfalls nach der Gründung einer gesamtschweizerischen Organisation wieder gestellt werden ${ }^{46}$. Für die Genfer ging es also um eine doppelte Anerkennung: Sowohl der Anschluss an die internationale Krebsforschung als auch die Subventionswürdigkeit durch den Bund konnten nur über den Umweg einer gesamtschweizerischen Organisation erreicht werden. Zugang zu wissenschaftlicher Erkenntnis, wissenschaftlicher Ruf und Beschaffung von Forschungsgeldern hing also nicht unwesentlich vom Erfolg der Gründung einer eidgenössischen Krebsorganisation ab. Es ist deshalb auch nicht verwunderlich, dass die wesentlichen Impulse und Vorarbeiten zur Gründung des «Schweizerischen Komitees für Krebsbekämpfung» von Genf ausgingen.

Die Schweizerische Vereinigung für Krebsforschung und -bekämpfung (SVK)

Dr. Schmid setzte sich daher bald mit dem Genfer Komitee in Verbindung und besprach mit den Herren Lardy, Odier und Duparc das Vorgehen zur Gründung eines schweizerischen Komitees zur Krebsforschung. Insbesondere wurde eine Liste aller in Frage kommenden Mitglieder ( «Gelehrte aus 
allen Teilen der Schweiz») aufgestellt und an diese die Einladung zur Gründung verschickt ${ }^{47}$.

Nach einigen überwundenen Schwierigkeiten bildete sich dann am 1.5. 1910 unter dem Vorsitz von Dr. Schmid das «Schweizerische Komitee für Krebsforschung». Erstes Ziel war, sich an der «internationalen Vereinigung für Krebsforschung» zu beteiligen. Zu diesem Zweck strebte das schweizerische Komitee die Bildung einer schweizerischen Gesellschaft an, "(...) die finanziell das schweizerische Komitee unterstützen \& ihm erlauben wird, das vorgenommene Ziel zu verfolgen.» Als potentielle Mitglieder kamen «(...) alle Ärzte \& die meisten Personen, die eine einflussreiche Stellung in der Schweiz einnehmen» in Frage ${ }^{48}$.

Im April 1912 erging deshalb ein Brief an die Öffentlichkeit, in dem für die Mitgliedschaft einer zu gründenden «schweizerischen Vereinigung für Krebsforschung» geworben wurde. In erster Linie sollte die Vereinigung die nötigen Mittel beschaffen, die für das Krebsprogramm des schweizerischen Komitees für Krebsbekämpfung (das als Vorstand der Vereinigung fungierte) gebraucht wurden ${ }^{49}$.

Allerdings verlief die Rekrutierung von Mitgliedern und Geldern nicht so, wie es sich zumindest Dr. Odier vorgestellt hatte. Von etwa 5000 Einladungen (an alle diplomierten Schweizer Ärzte) zum Beitritt zur Vereinigung wurden nur gerade 400 beantwortet. Ende 1913 waren nicht ganz 700 Mitglieder verzeichnet und 1914 stagnierte der Mitgliederbestand ${ }^{50}$ (wohl unter anderem wegen des Kriegsausbruchs, wie auch das Vorstandsprotokoll bemerkte ${ }^{51}$ ). In der Vorstandssitzung vom 6.12.1913 warf Odier dem Komitee vor, «(...) dass in den übrigen Kantonen viel zu wenig getan worden sei, sonst hätten wir in der S.V.K bereits eine Million Franken Vermögen.» ${ }^{52}$ Wegen dieser und anderer Differenzen scheint Odier 1915 aus dem Vorstand ausgetreten oder entlassen worden zu sein. Trotzdem liess er aber seine Kritik am fehlenden Engagement weiter verlauten. 1925 beklagte er sich:

«De tout ce que je viens de dire, on peut conclure que la lutte contre le cancer en Suisse n'a pas suscité l'intérêt que l'on était en droit d'attendre en faveur d'une œuvre comme celleci..» ${ }^{53}$

Wichtige Behinderungen machte er bei den Ärzten aus, die nur auf ein sicheres Mittel gegen Krebs warten würden, sich aber nicht in dieser Frage engagieren wollten, weil ihnen Zeit und Geld zu schade seien. Die meisten Ärzte würden nicht einmal die moralische Unterstützung und die Mithilfe an der Aufklärung der Patienten leisten, monierte er weiter. Alle seien nur an 
der Tuberkulose interessiert, bei der die Heilungschancen dank des medizinischen Fortschritts schon sehr gross und auch für weitere Kreise evident seien. Auch warf er der Medizin generell mangelnde Flexibilität vor. Hoffnungsvolle Forschungsansätze würden frühzeitig beerdigt :

«L'infaillibilité de la médecine «officielle` a enterré bien des hommes et bien des œuvres, et on pourrait la mettre en tête des causes qui, de tout temps, ont retardé les progrès des sciences médicales.» ${ }^{54}$

Der Fluch der Krebsforschung sei zudem, dass man noch zu wenig wisse und könne, um das Fach- und Laien-Publikum und das Geld anzulocken, ohne das man aber kaum zu neuen Erkenntnissen kommen könne.

Rosselet sieht die Rekrutierungsschwierigkeiten eher beim Individualismus, der Fehleinschätzung und wohl auch bei der Eitelkeit der Ärzte:

«Certains médecins en sont si convaincus (que nos malades soient bien soignés) que, recroquevillés sur un individualisme farouche, (...) comme sur la conscience du devoir accompli, ils ne veulent pas se laisser prendre dans une organisation (...). Ils ont tort.» ${ }^{55}$

Trotz der unerwartet schwachen Unterstützung durch das Fach- und Laienpublikum und durch den nur ehrenamtlich tätigen Vorstand entwickelte die SVK bald eine rege Tätigkeit.

Neben den oben erwähnten Mitgliedern der Genfer Société erklärten sich nach dem ersten, von der Genfer «Société» entworfenen und verschickten Aufruf zur Gründung einer gesamtschweizerischen Gesellschaft zur Krebsbekämpfung nur gerade 13 Ärzte bereit, sich ins Initiativkomitee aufnehmen zu lassen. Allerdings befanden sich darunter Persönlichkeiten wie Kocher, Kolle, Tavel oder Krönlein. Gemäss einer nachträglich von Hand ergänzten (undatierten) Liste des Initiativ-Komitees ${ }^{56}$ zählte das Komitee schliesslich nach einem weiteren Aufruf 38 Ärzte und Prominente. Davon kamen 10 aus Genf, 9 aus Bern, 7 aus Zürich, 4 aus Lausanne, 3 aus Basel, 2 aus Aarau und je 1 aus Neuenburg, St.Gallen und Münsterlingen (TG). Insgesamt darf man davon ausgehen, dass die Gründung einer Vereinigung von einem Grossteil der berühmten Schweizer Ärzte unterstützt wurde. Umso erstaunlicher ist die oben erwähnte Mühe, eine möglichst grosse Mitgliederzahl zu werben. Gründe dafür mögen die oben von Odier erwähnten und u.a. auch die Auslastung der Ärzte gewesen sein (siehe unten die wiederholte Absage von Rossier ${ }^{57}$ ).

Welche Persönlichkeiten des Initiativ-Komitees dann wirklich Mitglied der neugegründeten Vereinigung wurden, lässt sich auf Grund einer fehlenden zuverlässigen Mitgliederliste für 1910 nicht sagen. Lediglich die Zusam- 
mensetzung des Vorstandes ist bekannt. Ärzte aus den vier Universitäten Bern, Basel, Genf und Zürich stellten paritätisch den ersten, von der Gründungsversammlung gewählten Vorstand (das «engere Comité»), wenn man von Schmid, der ex officio Vorstandsmitglied war, absieht. Rossier (Lausanne) wurde zwar gewählt, lehnte aber Mitgliedschaft und Einsitz in den Vorstand $a b^{58}$. Das in den Statuten festgehaltene Ziel war, alle Universitätskantone im Vorstand repräsentiert zu haben. Es wurde 1913 mit der Aufstockung des Vorstandes von anfangs fünf auf nunmehr zwölf Mitglieder annähernd erreicht. Neben je zwei Ärzten aus Basel, Bern, Genf und Zürich wirkten auch je einer aus Lausanne und Neuenburg mit. Die Berner «Fraktion» stellte mit dem Vertreter des Gesundheitsamts und dem Kassier während Jahren die grösste kantonale Vertretung dar. Auch sonst war der «Berner Geist» überrepräsentiert: Nicht weniger als acht Vorstandsmitglieder der ersten zehn Vereinsjahre waren Schüler von Kocher gewesen. Etliche Vorstandsmitglieder hatten Unterricht bei anderen Berner Professoren (Langhans, Jadassohn, Stooss u.a.) genossen. Insgesamt hatte die Hälfte (13) aller Vorstandsmitglieder der ersten zehn Jahre vor oder während den Jahren 1910-1919 zwecks Forschung, Lehre oder Studium einen längeren Aufenthalt in Bern absolviert. Demgegenüber waren die Universitäten Basel (8 Mitglieder), Zürich (7), Genf (4), Lausanne (2) und Neuenburg (1 oder 2) deutlich weniger besucht. Nach dem Austritt Odiers aus dem Vorstand und dem Tod seines Nachfolgers Girard verlor Genf in der SKL zusätzlich an Bedeutung. Von den Genfer Mitgliedern des Initiativkomitees engagierte sich niemand mehr in der SVK. Die sowohl von der Genfer «Société» als auch vom Präsidenten der IVK anscheinend gewünschte Führungsrolle der Genfer Krebsbekämpfer ${ }^{59}$ war somit schon von Anfang an dem föderativen Gedanken geopfert worden. Weshalb dann aber Genf plötzlich ins Abseits geriet, bleibt eine offene Frage. Ob das Zerwürfnis mit Odier sich auf die anderen Genfer Forscher ausgewirkt hat oder Genf aus irgendwelchen Gründen forschungsmässig nicht auf der Höhe blieb, einen alternativen Weg zur SVK ging oder Genfs Interesse an der SVK einfach abgenommen hatte, müsste noch geklärt werden ${ }^{60}$. Zumindest ist festzuhalten, das die welschen Kantone in der Einrichtung von Institutionen zur Krebserforschung und -bekämpfung gesamtschweizerisch gesehen sehr aktiv blieben. Die Gründung des Genfer Radiuminstituts und der centres anticancéreux in Genf und Lausanne (1924) zeugen davon. Es darf somit angenommen werden, dass die Westschweiz forschungsmässig sicher weiterhin einen hohen Stand behielt, aber andere Akzente setzte als die SVK. Legte die SVK in den ersten 
Jahrzehnten den Schwerpunkt auf die Aufklärung, waren die Romands eher an Forschung und therapeutischer Praxis interessiert. Eine Rolle dürfte dabei u. a. die unterschiedliche wissenschaftliche Orientierung von Deutschund Welschschweizer Ärzten (und Wissenschaftlern allgemein) gespielt haben. Während die Ärzte der Romandie sich stets stärker an Frankreich anlehnten, richteten sich die Deutschschweizer vornehmlich nach Deutschland und Österreich aus. Es ist deshalb auch nicht verwunderlich, dass die Vorstandsmitglieder der SVK ihren wissenschaftlichen Horizont vornehmlich (aber nicht ausschliesslich) an deutschen Universitäten erweitert hatten, in erster Linie in Berlin (7 Mitglieder) und Wien (6). Aber auch München (4), Königsberg (4), Heidelberg (3), Leipzig, Halle, Göttingen, Würzburg, Frankfurt am Main, Breslau und Tübingen (je 1-2) waren besucht worden. Daneben waren auch in Paris (8) und London (5) Studienaufenthalte absolviert worden, allerdings meistens nur kurze.

Bis 1919 änderten sich sowohl das Profil als auch die Grösse des Vorstandes wenig. Alle medizinischen Vorstandsmitglieder waren mindestens teilweise in Forschung und Universität tätig, hatten zumeist eine nationale bis internationale Reputation auf den Gebieten Pathologie/pathologische Anatomie, Chirurgie, Hygiene/Epidemiologie, Gynäkologie und hatten sich schon vor ihrer Tätigkeit in der SVK wissenschaftlich mit Krebs auseinandergesetzt. Das wichtigste Gremium der SVK war also ein eigentliches Fachgremium, dessen Interesse am Krebs primär wissenschaftlich begründet war. Direktbetroffene oder karitativ tätige Kreise hatten daran keinen Anteil. Diese waren, wenn überhaupt, an der Basis, als gewöhnliche Mitglieder SVK zu finden.

Erst 1919/20, mit der erneuten Aufstockung des Vorstandes auf 18 Mitglieder fanden auch Praktiker aus Nicht-Universitätskantonen Eingang in das engere Komitee, allerdings meist solche mit akademischen Affinitäten.

Die SVK war also in ihren Anfängen weitgehend ein Gremium interessierter Mediziner, die, in den verschiedensten medizinischen Bereichen mit der Krebskrankheit konfrontiert, den im Ausland noch jungen und in der Schweiz noch kaum selbständigen Zweig Krebsforschung fördern wollten und zu diesem Zweck einerseits die weitgehende Spezialisierung in Forschung, Therapie und Pflege und andererseits die Aufklärung und Sensibilisierung der Bevölkerung betrieben (wie es in Artikel 2 der Gründungsstatuten auch zur Aufgabe gemacht wurde). 


\section{Die Krebsliga im historischen Kontext}

\section{Vergleich mit anderen gesamtschweizerischen Gesundheitsorganisationen}

Anders als beispielsweise die Tuberkuloseliga (1903 als «Schweizerische Zentralkommission gegen die Tuberkulose» gegründet ${ }^{61}$ ) oder die Alkoholliga (1902 gegründet, seit 1913 unter dem Namen «Schweizerische Zentralstelle zur Bekämpfung des Alkoholismus» (SAS) bekannt ${ }^{62}$ ), die entstanden sind, um die verschiedensten Aktivitäten zahlreicher lokal und regional tätiger Organisationen von der Pflege bis zur Forschung zu koordinieren und als Lobby die Interessen gegenüber Öffentlichkeit und Politik zu vertreten, war die SVK nicht Dachverband regionaler Institutionen. Sie konnte nicht auf ein schon vorhandenes Netz pflegerischer, fürsorgerischer oder forschungsorientierter Krebsorganisationen auf regionaler Ebene zurückgreifen, sondern musste sich eben dieses Netz zuerst schaffen. Die Krebsliga konnte und kann ihre Arbeit auch nicht auf eine Gesetzgebung, wie sie sowohl für Alkohol $\left(1887^{63}\right)$ als auch bald für Tuberkulose (1928 eidg. Tuberkulosegesetz ${ }^{64}$ ) bestand, abstützen. Anders auch als bei den «Volkskrankheiten» Alkoholismus und Tuberkulose fehlte es in der Bevölkerung am Bewusstsein um die Gefahr und Verbreitung der Krebskrankheit. Krebs war im Gegensatz zu Tuberkulose und Alkoholismus eine stille Krankheit, die sich kaum in der Öffentlichkeit manifestierte. Auch waren die Ursachen von Krebs noch kaum bekannt und dementsprechend Behandlungsmöglichkeiten und -erfolge noch recht bescheiden. Das im Rahmen der Hygienebewegung starke Interesse an präventiver Krankheitsbekämfpung, das sowohl bei der Alkohol- als auch bei der Tuberkulosebekämpfung einen prominenten Platz einnahm, konnte bei der Krebsbekämpfung nur rudimentär befriedigt werden, da, wie gesagt, die Krebskrankheit der Wissenschaft noch viele bedeutende Geheimnisse vorenthielt.

Es ist deshalb verständlich, dass der Kampf gegen den Krebs auf einem ganz anderen Niveau beginnen musste. Die am stärksten mit der Krankheit konfrontierten Personen, die Ärzte, (und hier speziell die Chirurgen) sahen die Notwendigkeit, gegen den Krebs vermehrt anzutreten, weil es mit dem zunehmenden Älterwerden der Bevölkerung und wegen anderen Faktoren für sie absehbar war, dass das Krebsrisiko dauernd steigen werde. Auch die Möglichkeiten, anhand von operativen Krebsbehandlungen einerseits den chirurgischen Erfahrungsschatz zu bereichern und die chirurgischen Techni- 
ken zu vervollkommnen und andererseits die Klinik (und dadurch die Chirurgie) weiter aufzuwerten, dürfte, zumindest im Hintergrund, eine Rolle gespielt haben. Sowohl Chirurgie wie mit Radioaktivität arbeitende Therapieformen erforderten eine Zentralisierung der Behandlung in den Räumen, in denen die dafür erforderlichen technischen Geräte und die Infrastruktur vorhanden waren, sowie eine Spezialisierung des wissenschaftlichen Personals, das mit den Apparaten umgehen konnte und die Techniken beherrschte. Eine durch Sozialmediziner propagierte Prävention wie bei Alkoholismus und Tuberkulose war kaum möglich, Laien waren im Kampf gegen den Krebs weitgehend zur Untätigkeit verurteilt. Also mussten die Experten die Initiative ergreifen und an zwei Fronten kämpfen. Die erste Front war die wissenschaftliche, die zweite die aufklärerische. An ersterer ging es darum, Wissen zu erarbeiten und Erfolge in der Behandlung zu erringen, an der zweiten, der Bevölkerung klar zu machen, dass Krebs eine Bedrohung der Volksgesundheit darstelle und dass nur bei Früherkennung eine einigermassen gute Heilungschance bestehe. Weiter sollte durch Aufklärung auch der Geldfluss angeregt werden, der für die aufwendige Forschung benötigt wurde. Die Koordination dieser Arbeit an zwei Fronten geschah mit Vorteil an einer zentralen Stelle. Erst wenn diese Vorarbeiten geleistet waren, konnte daran gedacht werden, Laien für die Tätigkeit gegen den Krebs zu mobilisieren und regional auf verschiedenen Ebenen von der Fürsorge bis zur Behandlung tätig zu werden.

Den erwähnten Gesundheitsorganisationen war gemeinsam, dass sie als wichtige Ansprechpartner des Bundes galten, wenn es darum ging, gesundheitspolitische Massnahmen und Entscheidungen zu treffen. Schon die organisatorische Nähe und die Personalverbindung mit dem Eidgenössischen Gesundheitsamt (EGA) durch dessen Direktoren Schmid und später Carrière ${ }^{65}$ deuten darauf hin. Auf der einen Seite konnte durch die Koordination innerhalb der einzelnen Organisationen eine zu stark föderalistische Problemlösung vermieden werden, auf der anderen Seite wurde der Bund durch die Abwälzung halbstaatlicher oder staatlicher Aufgaben auf private Institutionen entlastet (Milizsystem nicht nur in der Armee und der Politik, sondern auch in der staatlichen Verwaltung).

\section{Die SVK als Protagonistin medizinischen und gesellschaftlichen Wandels}

Die Gründung der Krebsliga steht im Kontext des Umbruches von der Hygienebewegung ${ }^{66}$ (die, in erster Linie epidemiologisch ausgerichtet, dar- 
auf abzielte, die Voraussetzungen von Krankheiten zu eliminieren) zum klinikorientierten, lokalistischen und technizistischen Gesundheitswesen. Eine vermehrte Weiterentwicklung von Spezialisierung und Professionalisierung des Medizinalwesens, Disziplinierung der Bevölkerung (regelmässige Kontrolle des eigenen Körpers, Gang zum Arzt, gesundheitskonformes Verhalten), Zentralisierung und Hierarchisierung der Gesundheitsversorgung sind Ausdruck dieses Prozesses. Dass die SVK Mitträgerin dieses Umbruches war, zeigen einerseits die Einordnungsversuche in die Hygienebewegung, um an deren Infrastruktur und dem Rückhalt in Bevölkerung und Politik teilzuhaben (manifest in den Diskussionen 1910 um die Teilnahme an der Hygienekonferenz in Dresden und 1917 um die Gründung einer allgemeinen Hygienegesellschaft, die angestrebt werden sollte, um der SVK mehr Gewicht zu verleihen, und schliesslich 1920 am Beitritt zur [inzwischen gegründeten] Schweizerischen Vereinigung für Hygiene, aber auch an der Mitgliedschaft etlicher Vorstandsmitglieder in Organisationen mit hygienischer Ausrichtung). Andererseits stellte die SVK im Sanitarisch-demographischen Wochenbulletin $(S D W B)^{67}$ klar,

«(...) dass ihre Aufgabe sei, die Schweizerärzte und auch die Bevölkerung immer wieder darauf hinzuweisen, dass das beste Heilmittel des Krebses zurzeit noch die Operation sei und dass vor allem eine frühzeitige Diagnose angestrebt werden müsse.»

Hygienische Massnahmen und Prävention im klassischen Sinne konnte die SVK also nicht geltend machen, einzig die Früherfassung konnte sie empfehlen und vor allem die Operation. Beide Möglichkeiten hängen von einem Arztbesuch ab. Massnahmen, die ein potentieller Patient ergreifen könnte, um Krebs zu verhindern, wurden, zumindest in der Anfangsphase der Krebsliga, kaum vorgeschlagen. Dafür wurde immer wieder die Notwendigkeit der ärztlichen Kontrolle betont. Die Behandlungsmöglichkeiten konnten und können zudem nur in entsprechend technisch ausgerüsteten Operationsräumen, meist in Kliniken, wahrgenommen werden. In ihren praktischen Anliegen kann die SVK deshalb nur insofern der hygienischen Bewegung zugeordnet werden, als sie versuchte, durch Aufklärung von Bevölkerung, Ärzten und Politikern die Volksgesundheit möglichst zu heben. In Bezug auf die Behandlung der Krankheit ging sie aber schon den direkten Weg in die Klinik ${ }^{68}$, in der der einzelne Patient nicht mehr dieselbe Verantwortung trägt wie sie ihm von der Hygienebewegung auferlegt wurde (indem sie ihn zur gesunden Lebensweise anhielt). Die Verantwortung reduzierte 
sich in der Klinik im wesentlichen darauf, sich dem Arzt und dem Personal anzuvertrauen und allfällige Weisungen genau zu befolgen.

Dass die SVK auch die Funktion hatte, die Interessen der wissenschaftlich ausgebildeten Fachärzte gegenüber nichtwissenschaftlichen Methoden oder dem medizinischen Konsens zuwiderlaufenden Auffassungen zu verteidigen, sollen folgende Beispiele belegen. Im $S D W B^{69}$ heisst es zum Zweck der SVK unter anderem: «Ferner soll Front gegen sogenannte Krebsheilmittel, die wertlos sind, gemacht werden.»

«Sogenannte» Krebsheilmittel, deren Heilerfolg mit wissenschaftlichen Methoden nicht nachgewiesen werden konnte, sollten also bekämpft werden. Wissenschaftlich überprüfbare Heilmittel dagegen wurden, auch wenn ihre Erfolgschancen minim waren, möglichst weiterentwickelt und erst bei Gewissheit der Unbrauchbarkeit abgesetzt.

Im Verlauf der ersten zehn Jahre sind namentlich zwei Fälle bekannt, in denen sich die SVK gegen Angriffe auf die von ihr propagierten Methoden und Massnahmen wehrte. Der erste Fall ist derjenige des von Neuchâtel aus tätigen John Shaw. Shaw vertrat die Ansicht, dass das Primat der operativen Behandlung von Krebs die Krebssterblichkeit nicht verhindere, sondern im Gegenteil erhöhe ${ }^{70}$. Anhand eidgenössischer Statistiken versuchte er zu beweisen, dass gerade bei den am häufigsten operierten Krebsen die Sterblichkeit dauernd zunehme. Krebs sei eine konstitutionelle Krankheit und sei nicht von lokaler Natur. Deshalb begünstige eine Operation die Disposition der Krankheit. Er empfahl zur Behandlung andere Methoden wie Elektrizität, ätherische Öle usw. Die Reaktionen im Vorstand der SVK waren dementsprechend heftig. In der Sitzung vom 6.11.1915 wurden Shaw Willkür, Fehlinterpretation, Verdrehung der Tatsachen und sogar «mystische Theorien» vorgeworfen. Schmid plädierte zwar dafür, dass man Shaws Aussagen zuerst überprüfen, die statistischen Angaben kontrollieren und durch eine eigene Statistik allenfalls falsifizieren solle. Dessenungeachtet wurde sofort eine Kampagne gegen Shaw eingeleitet, die zwar «die Ansichten Shaw's, nicht aber den Mann» treffen sollte. Immerhin attestierte man ihm, «nicht ein Schwindler im gewöhnlichen Sinne» zu sein. Aber als Arzt sei er in England von seinen Kollegen gehasst ${ }^{71}$.

Obwohl Shaws Ansichten vermutlich zur Bekämpfung des Krebses untauglich waren, unterliess es die SVK, dies wissenschaftlich zu untersuchen, sondern sprach ihm a priori die wissenschaftliche Haltbarkeit seiner Aussagen ab. 
Der zweite Fall ist derjenige des Dr. Aebly. Aebly kritisierte die von der SVK erstellte Brustkrebsstatistik, besonders, dass die Zahlen falsch interpretiert worden seien und Heilungserfolge durch Operation suggerierten, die nicht der Realität entsprächen. Im Vorstandsprotokoll steht darüber, dass Aeblys Artikel «nicht im Interesse der Krebsforschung und unserer Gesellschaft» liege. In einem Gegenartikel konterte dann de Quervain. Auch in diesem Fall ist die Argumentation eigenartig. Diesmal wurde nicht die Wissenschaftlichkeit ungeprüft abgesprochen, sondern die Kritik wurde als von der Linie der SVK abweichend charakterisiert. Explizit wurde das Interesse angesprochen, das zu verteidigen sei $^{72}$.

Die SVK ist also unter anderem als Element des anfangs beschriebenen Etablierungs- und Professionalisierungsprozesses zu verstehen. Inwieweit sie auch Akteurin des Disziplinierungsprozesses war, ist schwer abzuschätzen. Dazu müsste die Öffentlichkeitsarbeit unter diesem Aspekt analysiert und die Rezeption in der Bevölkerung untersucht werden ${ }^{73}$. Vermutlich war die Disziplinierungskraft im untersuchten Zeitraum noch gering, jedoch kam die Absicht, in dieser Richtung zu wirken, doch recht deutlich in den Merkblättern zum Ausdruck.

\section{Bibliographie}

a) Ungedruckte Quellen

Schweizerisches Bundesarchiv (SBA):

Mappe: Schweiz. Komitee für Krebsforschung. Sign.: 3300 (A), 1, 47

Archiv Schweizerische Krebsliga (SKLA):

Akten von Prof. Dr. Tavel 1909-1912. Sign.: A. 1.1.

Generalversammlungen, Protokolle und Unterlagen 1913-1960. Sign.: A. 3.1.

Protokolle der SVK 1910-1933. Sign.: A. 3.3.

b) Periodika

CSA (1871-1919): Correspondenzblatt für Schweizer Ärzte (Forts.: Schweizerische Medizinische Wochenschrift). Basel.

RMSR (1881ff.): Revue médicale de la Suisse Romande. Genf.

SDWB (1894-1917): Sanitarisch-Demographisches Wochenbulletin. Bern.

SMW (1920 ff.) : Schweizerische Medizinische Wochenschrift. Basel.

c) Gedruckte Quellen und Literatur

Ackerknecht, Erwin (1961): Die Auffassung des Krebses im Wandel der Zeiten (SA. aus: Oncologia 14/1961).

Alkoholgesetz (1987): 1887-1987: 100 Jahre Alkoholgesetz. o. O.

Bachmann, Ernst (1942): Die Tuberkulosebekämpfung in der Schweiz (SA. aus: Gegen die Tuberkulose, 1942). Bern. 
Bericht (1911): Bericht über die Tätigkeit der Schweizerischen Zentralkommission und der übrigen schweizerischen, kantonalen und kommunalen Vereinigungen zur Bekämpfung der Tuberkulose im Jahre 1910. Bern.

Bode, Ulrike (1979): Frühe histologische Krebsforschung in Deutschland, Frankreich und Österreich. Köln.

Dietrich, Albert (1936): Marksteine der Krebsforschung (SA. aus: Klinische Wochenschrift, 15. Jg. Nr. 37, 1936).

Di Nicola, Antonio/Bleiber, Brigitte (1991): Die Öffentlichkeitsarbeit der Schweizerischen Krebsliga 1910-1985. Diss. med. Bern.

Foucault, Michel (1976): Die Geburt der Klinik. Frankfurt/M., Berlin, Wien.

Labisch, Alfons (1986) : «Hygiene ist Moral-Moral ist Hygiene»-Soziale Disziplinierung durch Ärzte und Medizin. In: Sachse, Ch./Tennstedt, F. (Hgg.) (1986): Soziale Sicherheit und soziale Disziplinierung. Beiträge zu einer historischen Theorie der Sozialpolitik, Frankfurt/M., 265-285.

Mattmüller, Markus (1979) : Der Kampf gegen den Alkoholismus in der Schweiz. Ein unbekanntes Kapitel der Sozialgeschichte im 19. Jahrundert. (Helfen und Heilen 7). Bern/Wuppertal (Blaukreuzverlag).

Meerwein, Peter (1980): Der Krebspatient und sein Arzt im 19. Jahrhundert. Ursprünge der Pathologie? Zürich.

Mesmer, Beatrix (1982): Reinheit und Reinlichkeit. Bemerkungen zur Durchsetzung der häuslichen Hygiene in der Schweiz. In: Gesellschaft und Gesellschaften. Festschrift zum 65. Geburtstag von Prof. Dr. Ulrich Im Hof, hg. v. N. Bernard und Q. Reichen. Bern, 470-494.

Nager, Felix (1904): Bericht über die auswärtigen Untersuchungen der Tumorenstation vom 1. Januar 1900 bis 1. Januar 1904. Aus dem pathologischen Institut der Universität Zürich (SA. aus: CSA, 1904/9). Basel.

Odier, Robert (1907): La lutte contre le cancer à l'entrée du XXème siècle. Conférence faite à Genève le 15.4.1907. Genf.

Odier, Robert (1925): Contribution à l'étude du Cancer. L'Ornière de la médecine officielle. Genf.

Ribbert, Hugo (1911): Das Karzinom des Menschen. Sein Bau, sein Wachstum, seine Entstehung. Bonn.

Rosselet, Alfred (1934): L'organisation de la lutte contre le Cancer en Suisse. In: Organisation der Krebsbekämpfung (IV. Internationaler Radiologenkongress Zürich 1934, Band III), 185-191. Zürich.

Shaw, John (1915): Le Cancer en Suisse. La logique des faits. Neuenburg.

Sigerist, Henry E. (1960): On the history of medicine. New York.

Staehelin, Rudolf (1927): Die Bekämpfung der Tuberkulose und das neue Tuberkulosegesetz (SA. aus: Schweiz. Zs. f. Gesundheitspflege, 7, 1927). Zürich.

Theurillat, Michael (1982): Erkenntnisse und Forschungen über den Krebs 1870-1900 im Spiegel des «Correspondenzblatt für Schweizer Ärzte» (Diss. med. Basel). o. O.

Tuberkulose (1917): Die Tuberkulose und ihre Bekämpfung in der Schweiz. Sammlung von Aufsätzen (hg. von Schweiz. Zentralkomm. z. Bekämpfung d. Tuberkulose). Bern.

Wegelin, Carl (1959): Geschichte der Schweizerischen Nationalliga für Krebsbekämpfung und Krebsforschung 1910-1958 (Suppl. Oncologia 12, 1959). Basel.

Wunderlin, Dominik (1986): Die Antialkoholbewegung in der Schweiz (SA. aus: Alkohol im Volksleben). Marburg. 
Wyss, Max Oscar (1908): Zur Entstehung primärer Karzinome (Habil. Zürich) Leipzig.

Wyss, Max Oscar (1910): Über den gegenwärtigen Stand der Krebsforschung (SA. aus: CSA $1910 / 20)$.

\section{Anmerkungen}

1 Dieser Artikel beruht auf einer von der SKL finanziell unterstützten Seminararbeit in Schweizergeschichte bei Frau Prof. B. Mesmer, Historisches Institut der Uni Bern: Stefan Hächler (1991): «Örtliches Leiden». Die Gründungsgeschichte der Schweizerischen Krebsliga (SKL) (Typoskript).

2 WEGELIN (1959)

3 Die SKL änderte mehrmals ihren Namen, z. B. 1940 von «Schweizerische Vereinigung für Krebsbekämpfung» in «Schweizerische Nationalliga für Krebsbekämpfung» und 1967 in «Schweizerische Krebsliga».

4 SIGERIST $(1960,62)$

5 Ähnlich sieht es BODE $(1979,31)$ wenn sie schreibt, mit Müllers Werk «(...) war ein Anstoss zu weiteren Unternehmungen auf diesem Gebiet gegeben und gleichzeitig grobe Richtlinien für mögliche Forschungsarbeiten vorgelegt.»

6 Siehe dazu ACKERKNECHT $(1961,245)$

7 "Im grossen und ganzen aber erzielten die auf diesem Gebiet arbeitenden Wissenschaftler Europas weitgehende Einigung über die allgemeinen histologischen Bestandteile des Krebses. Differenzen gab es vor allem in der Beurteilung gutartiger oder bösartiger Geschwülste und bei der Zusammenstellung eines Systems, nach dem die histologisch unterschiedenen Arten der Geschwülste eingeteilt werden sollten (...). Nach den ersten Anfängen wurde die Zellhistologie nunmehr für die klinische Medizin interessant, und Kliniker wiederum interessierten sich für die mikroskopische Forschung (...). Diese Belebung der Forschung und die Verbreiterung ihrer Basis führte zu einer Unmenge neuer Ergebnisse und Erkenntnisse, die, trotz häufig nur minimalster Unterschiede zu vorherigen Forschungsergebnissen, veröffentlicht und verfochten wurden.» (BODE 1979, 32).

8 Klinik und Labor bildeten jetzt die zwei Zentren der Onkologie. Wobei oft das Labor zur Klinik wurde und umgekehrt. In diesem Sinne kann ACKERKNECHT $(1961,246)$ recht gegeben werden, wenn er schreibt: «Nach Virchow ist die Onkologie vor allem experimentell geworden.»

9 Vgl. DIETRICH $(1936,1297)$

10 DIETRICH $(1936,1298)$

11 Krebsbehandlung durch Röntgenstrahlen erstmals 1899 durch T.Sjögren (ACKERKNECHT 1961, 243).

12 Wyss (1874-1956) stammte aus einer Ärztefamilie (Sohn des bekannten Hygienikers und Pädiaters Oskar Wyss), studierte Medizin in Zürich und Berlin, dissertierte 1900 bei Krönlein, habilitierte (nach Assistenztätigkeit bei Krönlein, diversen Auslandaufenthalten und nach sechs Jahren eigener Praxis in Zürich) 1908 in Zürich mit dem Thema «Zur Entstehung primärer Carcinome». Er vertrat darin die Theorie Ribberts, die besagt, dass «(...) das Carcinom (...) aus Epithelzellen des Körpers [entsteht], die ausgeschaltet wurden, 
und die nun auf eigene Faust schrankenlos auf Kosten des Körpers weiterwachsen» (Ribbert, zit. nach WYSS 1908, 3). Wyss war ab 1912 über 30 Jahre lang leitender Arzt des Spitals Bethanien und ab 1917 zudem Arzt der Schweizerischen Rückversicherung.

13 WYSS $(1910,2)$

14 «Die Schweiz ist eines von denjenigen Ländern, welche die grösste Zahl von Krebstodesfällen aufweisen (...)» (aus einer öffentlichen Einladung zum Beitritt in die SVK vom April 1912, SKLA A. 1.1). Laut WYSS 1910 wies die Schweiz im Vergleich zur westlichen Welt die weitaus grösste Krebssterblichkeit auf. In Europa z. B. war die höchste Sterberate um 1900 nicht ganz ein Promille, in der Schweiz hingegen 1,32 Promille. Auch starben in der Schweiz im Gegensatz zu Resteuropa mehr Männer als Frauen an Krebs. Wyss sah auf Grund der Statistiken folgende möglichen Erklärungsansätze: Naturräumlichkeit, Lebensgewohnheiten, ethnische Disponibilitäten, Umwelteinflüsse.

15 Krebsspezialisten im eigentlichen Sinn des Wortes gab es auch international noch sehr wenige, hingegen gab es Ärzte aus den verschiedensten medizinischen Teilbereichen, die sich innerhalb ihres Fachgebietes intensiv mit Krebs auseinandersetzten.

16 Einen Überblick über Art, Qualität und Bedeutung dieser Artikel gibt THEURILLAT (1982).

17 Siehe THEURILLAT $(1982,139)$

18 Die Durchsicht der RMSR bestätigt diese Aussage. In den kontrollierten Jahrgängen 1905-1908 finden sich fast in jeder Nummer Artikel über die Erforschung und Behandlung von Krebs.

19 NAGER $(1904,1)$

20 ROSSELET $(1934,185)$

21 Robert Odier (1864-1928) promovierte 1901 mit einer Dissertation über Nervenzellen. Ab 1907 publizierte er verschiedentlich über Krebs. Er leitete mehrere Jahre das «Institut pour l'étude des maladies cancereuses», war PD an der Uni Genf und wurde 1913 Ehrenpräsident der Krebskonferenz in Brüssel.

22 ODIER $(1907,5)$

23 ODIER $(1907,4)$

24. ODIER $(1907,3)$

25 ODIER $(1907,11 \mathrm{f}$.)

26 ODIER $(1907,29)$

27 Erster Präsident war Dr. Edmond Lardy (s. Anm. 35).

28 Brief des Internationalen Komitees für Krebsforschung an den Präsidenten des Schweizerischen Gesundheitsamtes vom 1.12.1908, SBA 3300 (A), 1/47; ODIER (1925), 8; Brief von Dr. Odier an Dr. Schmid (Schweiz. Gesundheitsamt) vom 6.12.1908, SBA 3300 (A), $1 / 47$.

29 Art. 2 der «Statuts de la Société pour la lutte contre le cancer» (o. J.), SBA 3300 (A) 1/47.

30 ODIER $(1925,8)$

31 Brief von Dr. Odier an Prof. Tavel vom 25.1.1910, SKLA A. 1.1; ODIER $(1925,8)$.

32 ODIER $(1925,9)$. So z. B. Prof. O. Beuttner, Direktor der Maternité in Genf, der öffentliche Vorträge über frauenspezifische Krebskrankheiten hielt (ODIER, 1925, 9).

33 Leider konnte über die Trägerschaft, die Organisation und die Finanzierung der «Société» und des «Institut» in nützlicher Frist nichts genaueres in Erfahrung gebracht werden. Diesbezügliche Hinweise nimmt der Autor gerne entgegen. 
34 Brief der Intern. Vereinigung für Krebsforschung an Dir. Schmid von 1.12. 1908, SBA 3300 (A) $1 / 47$.

35 Dr. Edmund Lardy (1859-1935) von Neuenburg, war 2 Jahre Militärarzt in der Türkei, seit 1907 Mitglied der eidg. Medizinalprüfungskommission und wurde 1908 PD an der med. Fakultät der Uni Genf.

36 Siehe Anm. 21.

37 Dr. Charles Bergalonne (1873-1917) von Genf, war Chefchirurg an der chirurg. Klinik in Genf und während des Krieges Militärarzt in Frankreich.

38 Prof. Dr. Adolphe d'Espine (1846-1930) von Genf, war seit 1876 Prof. für interne Pathologie an der Uni Genf, seit 1878 Mitglied der schweiz. Ärztekommission, 1910 wurde er zum Leiter der Kinderklinik ernannt und war eine Zeitlang Präsident des IKRK.

39 Prof.Dr. Charles Girard (1850-1916) von St-Imier, war oP an der chirurgischen Klinik in Genf, vorher aoP am Berner Inselspital.

40 Dr. Jean Louis Reverdin (1842-1929) von Genf, war seit 1908 Prof. an der chirurg. Poliklinik, führte daneben mit seinem Cousin Auguste Reverdin in Carouge eine Privatklinik. Reverdin leitete während 30 Jahren die «Revue médicale de la Suisse Romande» (RMSR).

41 Prof. Dr. Léon Massol (1839-1909) begann als Ingenieur mit 40 Jahren das Studium der Bakteriologie am Institut Pasteur in Paris, wozu er vermutlich durch die enge Freundschaft mit J. L. Reverdin angeregt wurde. Vor seiner Berufung als Prof. für Bakteriologie 1900 war er Direktor des städtischen Genfer Bakteriologischen Labors.

42 Dr. Friedrich Schmid (1850-1916) von Meikirch (BE), war praktischer Arzt bevor er 1889 Sanitätsreferent des eidgenössischen Departement des Innern und 1893 erster Direktor des neu gegründeten Schweizerischen Gesundheitsamtes (nachmalig BAG) wurde. Hier entwickelte er rege Aktivitäten namentlich in den Gebieten Seuchenbekämpfung, Lebensmittelgesetzgebung, Landespharmakopöe, Medizinalprüfungswesen.

43 Brief des Internationalen Komitees für Krebsforschung an den Präsidenten des Schweizerischen Gesundheitsamtes vom 1.12.1908, SBA 3300 (A), 1/47.

44 Brief der «Société pour la lutte contre le Cancer» an den Präsidenten des Schweizerischen Gesundheitsamtes vom 6.12.1908, SBA 3300 (A), $1 / 47$.

45 Vermutlich ist das «Institut de l'étude des maladies cancéreuses» gemeint.

46 Brief der Bundeskanzlei an das Komitee «de l'Institut anticancereux à Genève» vom 9.3.1909, SKLA A. 1.1.

47 Rundbrief des Präsidenten des Schweizerischen Gesundheitsamtes an potentielle Mitglieder vom 8.10.1909, SBA 3300 (A) 1/47.

48 SKLA A. 1.1.

49 Offener Brief des schweizerischen Komitees für Krebsbekämpfung vom April 1912, SKLA, A. 1.1.

50 Siehe Abb. 2 in DI NICOLA/BLEIBER (1991)

51 Protokolle 1910-1915, 12, 14, 46.

52 Protokolle 1910-1915, 37.

53 ODIER $(1925,10)$

54 ODIER $(1925,7)$

55 ROSSELET $(1934,185)$ 
56 SKLA A 1.1; der Eintrag resultiert wahrscheinlich aus einer zweiten, die Unabhängigkeit der neuzugründenden Gesellschaft von der Genfer «Société» betonenden Einladung von Dr. Schmid (9.10.1909, SBA 3300 (a) 1/47).

57 Guillaume Rossier (1864-1928) war seit 1906 Prof. für Geburtshilfe und Gynäkologie an der Uni Lausanne. Er war als guter Operateur und Hebammenausbildner bekannt. Mit C. Roux und L. Bouget arbeitete er an der RMSR.

58 Protokolle der Vorstandssitzungen vom 3.6. 1910 und 28.2.1911 (SKLA a 3.3). WEGELIN (1959) erwähnt die Absage Rossiers nicht und sieht ihn fälschlicherweise als Mitglied des Vorstandes, ebenso ein Artikel im Sanitarisch-demographischen Wochenblatt vom 25.4.1914: «Schweizerische Vereinigung für Krebsbekämpfung. 1. Gründung und bisherige Entwicklung».

59 Czerny bittet Schmid in einem Brief vom 1.12.1908, die Gründung eines schweizerischen Komitees zur Krebsbekämpfung «... mit Mitgliedern aus allen Teilen des Landes, insbesondere aber auch mit den genannten Herren des Genfer Komitees, in die Wege zu leiten ...» (SBA 3300 (A) 1/47).

60 Allerdings weist eine Mitgliederliste aus dem SDWB von 1916 noch etliche Mitglieder der Société auf, unter anderem auch Dr. R. Odier. Auch andere Genfer und Waadtländer Ärzte waren weiterhin Mitglieder, traten aber durch ihr Engagement nicht in Erscheinung.

61 Vgl. z.B. BERICHT (1911), TUBERKULOSE (1917), STAEHELIN (1927), BACHMANN (1942). Ähnlich wie bei der Krebsliga hing bei der Tuberkuloseliga die Gründung mit dem Entstehen einer internationalen Vereinigung zusammen. Der Direktor des EGA, Schmid, war auch hier «Geburtshelfer».

62 Den Kampf gegen den Alkoholismus in der Schweiz schildern z. B. MATTMÜLLER (1979) und WUNDERLIN (1986).

63 Siehe ALKOHOLGESETZ (1987).

$64 \mathrm{Zu}$ Beginn des Jahrhunderts erliessen etliche Kantone Bestimmungen zur Bekämpfung der Tuberkulose, so z. B. Graubünden 1902, Zürich 1907, Bern 1908 usw. Auf eidg. Ebene existierte seit 1872 im Rahmen des Viehseuchengesetzes die Möglichkeit, gegen Rindertuberkulose vorzugehen. Die Verordnung betr. Verkehr mit Lebensmitteln von 1909/1914 erlaubte Massnahmen zur Verhütung der Tuberkulose durch Lebensmittel. Daneben gab es auf Bundesebene noch andere, indirektere Massnahmen, die die Verbreitung von Tuberkulose verhindern sollten. Vgl. dazu auch S. 46-53 in TUBERKULOSE (1917). Zum Tuberkulosegesetz siehe BACHMANN (1942).

65 Henri Carrière (1875-1941) von Dardagny (GE), wurde in Nîmes geboren, studierte Medizin in Genf und Paris, praktizierte danach im Waadtland. 1894 wurde er als medizinischer Adjunkt ins EGA berufen. 1916 wurde er als Nachfolger Schmids Direktor des EGA, bis er 1937 von seinem Amt zurücktrat. Seine Interessenschwerpunkte waren Tuberkulose, Lebensmittel- und Arzneikontrolle und Krebs.

66 Zur Hygienebewegung vgl. z. B. LABISCH (1986) und für die Schweiz MESMER (1982).

67 Nr. 15, 1914

68 Vgl. dazu FOUCAULT (1976).

69 Nr. 15, 1914

70 z. B. SHAW (1915)

71 SKLA A. 3.3 
72 Aeblys Artikel und de Quervains Antwort finden sich in: SMW 53/1920, 1178-1183. Im Gegensatz zur Diskussion in der SVK äussert sich de Quervain sachlich und fair zu den Vorwürfen von Aebly.

73 Zur Öffentlichkeitsarbeit siehe DI NICOLA/BLEIBER (1991)

$74 \quad$ SKLA A 3.3

\section{Stefan Hächler}

Medizinhistorisches Institut

Bühlstrasse 26

CH-3012 Bern 